\title{
Metasurface for Controlling Polarization of Scattered EM Waves
}

\author{
Mohammad Alibakhshikenari ${ }^{1 *}$, Bal S. Virdee ${ }^{2}$, Chan H. See ${ }^{3,4}$, Raed A. Abd-Alhameed ${ }^{5}$, Francisco \\ Falcone $^{6}$, and Ernesto Limiti ${ }^{1}$ \\ ${ }^{1}$ Electronic Engineering Department, University of Rome "Tor Vergata", Rome, ITALY \\ ${ }^{2}$ London Metropolitan University, Center for Communications Technology \& Mathematics, London, UK \\ ${ }^{3}$ School of Engineering \& the Built Environment, Edinburgh Napier University, Edinburgh, UK \\ ${ }^{4}$ School of Engineering, University of Bolton, Bolton, UK \\ ${ }^{5}$ Faculty of Engineering and Informatics, University of Bradford, Bradford, UK \\ ${ }^{6}$ Electrical and Electronic Engineering Department, Public University of Navarre, Pamplona, SPAIN \\ *alibakhshikenari@ing.uniroma2.it
}

\begin{abstract}
A unique metasurface is presented that can be used to control the polarization of electromagnetic waves. The dual band metasurface is realized by stacking an array of multiple concentric circular rings. By rotating each array with respect to each other, we can manipulate its impedance at distinct frequencies. The versatility of the proposed metasurface is numerically verified.
\end{abstract}

Keywords - Tensor impedance metasurface, electromagnetic (EM) waves, antenna applications, cascaded layers, impedance-matched metasurface, multiple concentric circular rings, multi-conductor transmission (MCTL), circular polarization selective surface (CPSS).

\section{INTRODUCTION}

Impedance matching is essential in RF systems to maximize power transfer from the signal source to the load [1]. Unfortunately, trade-off between the matching bandwidth and matching efficiency is imposed by the Bode-Fano criterion [2][3]. The implication of this is significant for the system bandwidth especially when the load $\left(Z_{L}\right)$ is highly dispersive, and when the impedance of the generator load $\left(Z_{g}\right)$ is higher than $Z_{L}$. Such a situation arises when small antennas are matched for communications and radars [1]. In this case when a pulse of short duration is transmitted it is seriously distorted as well as the associated group delay. We can however optimize the transmission efficiency, $\eta$, for a given generator impedance, $Z_{g}$, by discarding the impedance matching network for a suboptimal matching for a given load, $Z_{L}$.

Overcoming the matching constraints have been previously explored using active matching network realized with non-Foster reactive elements. Such matching networks have also been proposed to enhance the bandwidth of small antennas [4] and cloaking devices [5]. The downfall with this method is increase in the internal noise of the matching network as well the likely hood for the network to become unstable [6]. Furthermore, although non-Foster matching networks can counteract the effects of reactive loads, however they cannot be used to match resistive loads.

To compensate the effects of suboptimal antenna matching requires the use of artificial surfaces referred to as metasurfaces that can be used to overcome distortion of electromagnetic (EM) waves interacting with two media of different material characteristics [7].

Metasurfaces have gained popularity for manipulating various characteristics EM waves as they are relatively easy to construct and have a low profile. Metasurfaces are constructed using subwavelength sized scattering elements referred usually as unit-cells that are arranged in arrays. The unit-cells interact with the electromagnetic-field to affect its scattering properties such that an incident wave is precisely controlled. The unit-cells constituting the metasurface can be tuned to determine specific phase or amplitude profile of the EM-wave propagating over the metasurface to engineer specific scattering properties including the polarization state of the scattered EM-waves. This type of manipulation is of use in numerous microwave and optical applications [8].

This paper presents impedance-matched metasurface structure that can be used to control the polarization of scattered EM-waves in terms of its selectivity or rotation. With the proposed metasurface specific surface impedances can be realize at two different medias. This is accomplished by cascading layers of arrays of numerous concentric circular rings. Adjacent layers are rotated with respect to each another, and the angle of this rotation is used to determine the structure's specific property.

\section{SYNTHESIS OF IMPEDANCE MATCHED METASURFACES}

Investigated here is a new technique to accomplish impedance matching for pulses of short duration. Fig. 1 shows a pulsed source that is connected to a load through a transmission-line, where $Z_{L}=R_{L}$. Note, $Z_{c}$ is the characteristic impedance of the transmission-line. Here, the impedances $Z_{c}, R_{g}$, and $Z_{L}$, are considered nondispersive. Under the condition when $Z_{c}=R_{g}$, the power delivered from the source to the transmission-line is maximum. If the load impedance is not matched to the transmission-line's characteristic impedance, i.e. $Z_{L} \neq Z_{c}$, the transmitted pulse will be reflected from the load. We can prevent reflections if we could, while the pulse is propagating on the transmission-line, switch the 
transmission-line impedance to be $Z_{c}=Z_{L}$. Hence, optimum power transfer will be achieved in this case.

This scenario suggests that the possibility of determining a switching scheme that results in significantly improved matching than that achieved by conventional time-invariant impedance matching networks. Furthermore, the proposed suggestion is not subject to the Bode-Fano restriction.

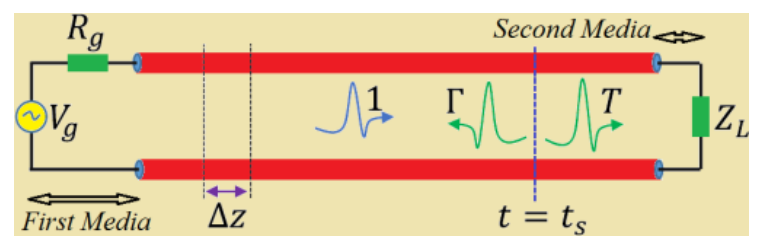

(a)

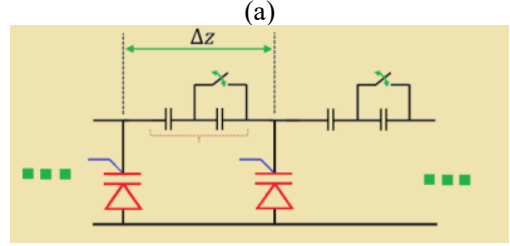

(b)

Fig.1. (a) Switched transmission-line, and (b) potential switched transmission-line implemented using a discrete periodic structure of parallel varactor diodes and switched series capacitors.

The transmission-line shown in Fig.1 can be implemented using metasurfaces that is impedance matched. The metasurface is realized using circularly polarized selective structure (CPSS) applied between two different media. Impedance-matched CPSS is realized by stacking together five metasurfaces separated from each other by $2 \mathrm{~mm}$ as illustrated in Fig. 2. The metamaterial layer comprises arrays of multiple copper based concentric circular rings whereby the layers are rotated with respect to each other.

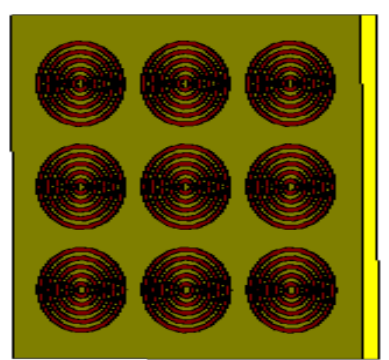

(a)

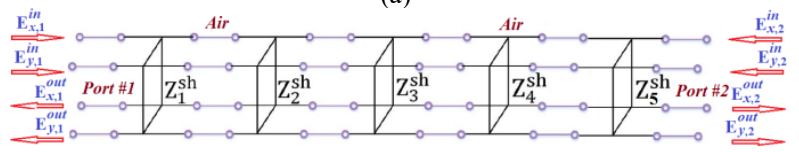

(b)

Fig.2. (a) Layout of metasurface layer which is fabricated on a $0.2 \mathrm{~mm}$ thick substrate, and (b) its equivalent multi-conductor transmission-line model.

The gap between the concentric circular rings plays the role of the series left-handed capacitance $\left(C_{L}\right)$, which is shown in Fig.1(b). The periodicity of the unit-cell is $2 \mathrm{~mm}$. The required impedances were physically mapped by setting the geometries of the rings, from outer to inner rings, as $2.5 \times 2.25 \mathrm{~mm}^{2}, 2 \times 1.75 \mathrm{~mm}^{2}, 1.5 \times 1.25 \mathrm{~mm}^{2}$, $1 \times 0.75 \mathrm{~mm}^{2}$, and $0.5 \times 0.25 \mathrm{~mm}^{2}$ for all layers. The widths of the rings are fixed to $0.25 \mathrm{~mm}$.
The insertion-loss (IL) for right-hand circular polarization (RHCP) and the Return-loss (RL) for left-hand circular polarization (LHCP) for the physical layers are shown in Fig. 2. It is noted that the IL at the two operating frequencies are all below 1-dB indicating field transmission efficiency greater than $90 \%$. The axial ratio at the design frequencies are near unity for the transmitted RHCP and reflected LHCP.

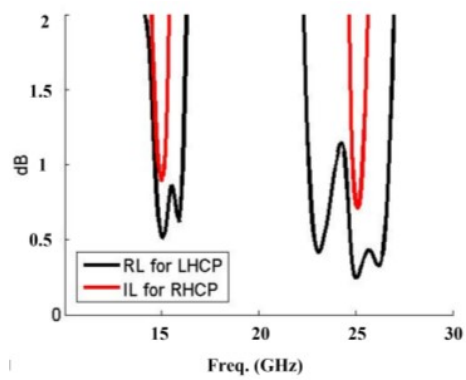

Fig. 2 The IL of RHCP (red) and RL of LHCP (black).

\section{CONCLUSION}

A technique is presented to accomplish dual band operation using a metasurface that can control the polarization of scattered EM-waves. This was obtained by stacking together layers with arrays of multiple concentric circular rings. The properties of the proposed dual band circularly polarized selective structure have been numerically verified. It exhibits IL of RHCP and RL of LHCP lower than $1-\mathrm{dB}$ at $15 \mathrm{GHz}$ and $25 \mathrm{GHz}$ with near unity axialratio.

\section{ACKNOWLEDGMENTS}

This work is partially supported by innovation programme under grant agreement H2020-MSCA-ITN2016 SECRET-722424 and the financial support from the UK Engineering and Physical Sciences Research Council (EPSRC) under grant EP/E0/22936/1.

\section{REFERENCES}

[1] Amir Shlivinski, and Yakir Hadad, "Beyond the Bode-Fano Bound: Wideband Impedance Matching for Short Pulses Using Temporal Switching of Transmission-Line Parameters", Physical Review Letters 121, 204301 (2018).

[2] O. Acher, J. M. L. Bernard, P. Marchal, A. Bardaine, and F. Levassorta, Fundamental constraints on the performance of broadband ultrasonic matching structures and absorbers, J. Acoust. Soc. Am. 125, 1995 (2009).

[3] F. Monticone and A. Alú, Invisibility exposed: Physical bounds on passive cloaking, Optica 3, 718 (2016).

[4] S. E. Sussman-Fort and R. M. Rudish, Non-Foster impedance matching of electrically-small antennas, IEEE Trans. Antennas Propag. 57, 2230 (2009).

[5] P.-Y. Chen, C. Argyropoulos, and A. Alú, Broadening the Cloaking Bandwidth with Non-Foster Metasurfaces, Phys. Rev. Lett. 111, 233001 (2013).

[6] S. D. Stearns, Non-Foster circuits and stability theory, in Proceedings of the International Symposium on Antennas and Propagation (APSURSI), IEEE, 2011.

[7] Minseok Kim and George V. Eleftheriades, "Dual-band chiral metasurfaces", 2017 IEEE International Symposium on Antennas and Propagation \& USNC/URSI National Radio Science Meeting, San Diego, CA, USA, 9-14 July 2017.

[8] Minseok Kim, and George V. Eleftheriades, "Design and Demonstration of Impedance-matched Dual-band Chiral Metasurfaces", Scientific Reports, (2018) 8:3449. 